\title{
OBSERVATIONS OF THE LEONIDS IN CENTRAL ASIA
}

\author{
P.B.BABADZHANOV \\ Institute of Astrophysics of the Tajik Academy of Sciences \\ Dushanbe 734042 \\ Tajikstan
}

Observations in Central Asia in 1965-1966 by both photographic and radar methods allowed a determination of the radiants and orbits of Leonids (Babadzhanov and Getman 1970). Photographs showed that meteoroids undergo quasi-continuous fragmentation (QCF) in the Earth's atmosphere. Taking account of QCF, the density of the Leonid meteoroids were found to lie between 1 and $4 \mathrm{gcm}^{-3}$ the average being $2 \mathrm{gcm}^{-3}$ (Babadzhanov 1994), in agreement with the density range of between 0.2 and $6 \mathrm{gcm}^{-3}$ given by Maas et al (1990) for dust grains from comet P/1 Halley, with values below 0.6 being rare. Further, the icy grains have a density of about $1 \mathrm{gcm}^{-3}$ while silicate grains have a mean density 2.5 times higher.

The following observations are planned during the activity period of the Leonids in 1997-99 from the Institute of Astrophysics, Dushanbe, Tajikstan. Photographic observations using 8 cameras $(F=750 \mathrm{mmD}: F=1: 3.5)$ equiped with high quality Zeiss Distagon fish-eye objectives taking imultaneous exposiures $\left(5.6 \times 10^{-4} s\right)$ and also two-station TV observations.

Observations of the Leonids are also planned in the Scientific -Technical Centre, Ashgabad, Turkmenistan by the radar system Cyclone with 4 antennas directed to the North, East, South and West, and by wide- angle $1 m$ telescope $(D: F=1: 1.8)$ equipped with a TV-camera permitting recording of Leonid meteors down to a magnitude of 11 .

\section{References}

Babadzhanov, P.B., (1990) Density of Meteoroids and their Mass Influx on Earth Asteroids, Comets, Meteors 1999 eds Milani A. Di Martino M. Cellino A. Kluwer Academic Publishers, 45-54

Babadzhanov, P.B. and Getman, T.I., (1970), Bull. Inst. Ast. Tajik Acad. Sci.,53, 3-6

Maas, D., Krueger, F.R. and Kissel, J. (1990), Asteroids, Comets, Meteors III, eds Lagerkvist, C.-I., Rickman, H., Lindblad, B.A. and Lindgren, M., Uppsala Universitet Reprocentralen, 389-392 